\title{
Editorial for Weed Science, Volume 64
}

\author{
William K. Vencill*
}

Weed Science continues to be one of the premier journals in the discipline and in the agronomic sciences. The Editorial Board and the staff strive to improve the efficiency of the publication process and improve the scope of the journal. Weed Science seeks to publish more reviews and symposium articles. This year, Weed Science will publish a special issue of Weed Science from presenters at the WSSA Herbicide Resistance Summit II.

For the 12 mo preceding September 1, 2015, 211 manuscripts were submitted to Weed Science, compared to 208 in the previous 12 -mo period. Of the manuscripts accepted, 44.3\% were accepted, compared to $40.8 \%$ the previous year. The time from submission to first decision was $44 \mathrm{~d}$ this past year compared to $45 \mathrm{~d}$ for the previous year.

Table 1 shows the Impact Factor and Article Influence Score for Weed Science and other related journals for 2014. The impact factor for Weed Science increased from 1.687 to 1.870 this past reporting cycle, whereas the 5-yr impact factor increased from to 1.928 from 1.835. I believe the latest impact factor and the fiveyear impact factor are the best the journal has ever had.

For the year preceding September 1, 2015, the journal depended on 283 unique reviewers from 34 countries for peer review. The excellent reputation of the journal would not be possible without the cutting-edge research submitted by authors and the diligent work of the editorial board and the hundreds of referred reviews that are submitted annually. I wish to thank the members of the editorial board and reviewers for 2015.

Table 1. Impact factor of Weed Science and comparable journals in 2014 (2012 Journal Citation Report 2015).

\begin{tabular}{lcc}
\hline Journal & $\begin{array}{c}\text { 2014 impact } \\
\text { factor }\end{array}$ & $\begin{array}{c}\text { Article influence } \\
\text { score }^{\mathrm{a}}\end{array}$ \\
\hline Weed Science & 1.870 & 0.657 \\
Weed Research & 1.687 & 0.538 \\
Crop Protection & 1.493 & 0.449 \\
Pest Management Science & 2.694 & 0.796 \\
Crop Science & 1.575 & 0.515 \\
\hline
\end{tabular}

${ }^{a}$ Article influence score takes into account the impact factor of the journals doing the citing.

DOI: 10.1614/WS-D-15-Editorial.1

* Professor and Editor, Department of Crop and Soil Sciences, University of Georgia, Athens, GA 30602. Corresponding author's E-mail: wvencill@uga.edu

\section{Editorial Board 2015}

Muthukumar V. Bagavathiannan

Bhagirath Chauhan

Sharon Clay

Adam Davis

Franck E. Dayan

J. Anita Dille

Timothy L. Grey

Amy Lawton-Rauh

John L. Lindquist

Vijay Nandula

Chris Preston

Dean Riechers

Hilary Sandler

Steven Seefeldt

Patrick J. Tranel

Theodore Webster

Martin M. Williams, II

\section{Weed Science Reviewers 2015}

Seth Abugho

Steve Adkins

Kassim Al-Khatib

Phil S. Allen

James E. Altland

Pedro Luis da Costa Aguiar Alves

Lars W. J. Anderson

Christian Andreasen

Andre Andres

Renee S. Arias

Gregory R. Armel

Jamshid Ashigh

Valentina Atanackovic

Carol Auer

Albert O. Ayeni

Muthukumar V. Bagavathiannan

Rodrigo Baggio

Elizabeth Baggs

Daniel A. Ball

Sanjeev K. Bangarwa

Mohammad T. Bararpour

Mary Barbercheck

John Barbur

Jacob Barney

Michael Barrett

Chhandak Basu 
Diego Batlla

Todd A. Baughman

Hugh J. Beckie

Roland Beffa

Michael Scott Bell

Robin R. Bellinder

Regina Belz

Roberto Benech-Arnold

Diane Lyse Benoit

Stafano Benvenuti

Therese Berge

Mark Lee Bernards

Thierry Eric Besancon

Prasanta Bhowmik

Henrique von Hertwig Bittencourt

Thomas Bjorkman

Jason K. Blackburn

Robert E. Blackshaw

Amy C. Blair

Walter Bleeker

David C. Blouin

Chris M. Boerboom

Jason Bond

George Botha

Peter Boutsalis

Nathan S. Boyd

Rick A. Boydston

Kevin W. Bradley

Daniel Brainard

Lars Olav Brandsæter

Inge Broer

Chad L. Brommer

James T. Brosnan

Nilda R. Burgos

Ian C. Burke

James D. Burton

Roberto Busi

John Dukes Byrd

Ana Caicedo

Ragan Callaway

Edinalvo Camargo

Charles Cantrell

John Cardina

Luke Thomas Case

Jane Catford

Jason Cathcart

Demosthenis Chachalis

Gurinderbir S. Chahal

Rakesh S. Chandran

Lindsay Chaney

Raghavan Charudattan

Carlene A. Chase

Jun Chen

Nick E. Christians

Michael J. Christoffers

Pedro Jacob Christoffoleti

Benjamin Clasen
Daniel Clements

Daniel C. Cloutier

Vanessa Coats

Ronald Collins

Jed B. Colquhoun

Amy Concilio

Jeffery Sam Conn

Warren Conway

Daniel Cook

Michael C. Cox

Nancy G. Creamer

Roberto J. Crespo

Hugo Cruz-Hipolito

A. Stanley Culpepper

William Stevenson Curran

John Cussans

Mark Andrew Czarnota

Caleb D. Dalley

Christos Damalas

Henri Darmency

T. K. Das

Joseph Dauer

Vince M. Davis

Hans de Kroon

Rafael Alberto De Prado Amian

Ignacio Dellaferrera

Christophe Delye

Rafael DePrado

Jeffrey F. Derr

Julia Detering

Giovanni Dinelli

Joseph M. DiTomaso

Katrina M. Dlugosch

Douglas J. Doohan

Peter A. Dotray

John Doudna

Emily B. M. Drummond

Susan Dudley

Stephen Duke

Ryan James Edwards

John Franklin Egan

Hanan Eizenberg

Ilias George Eleftherohorinos

Mohamed M. El-Hawary

Norman C. Ellstrand

Jonathan E. Ephrath

Dieter Ernst

Thomas William Eubank

Wesley J. Everman

Robert Faden

Muhammad Farooq

Brandon Fast

Joel Felix

Steven A. Fennimore

Diego Omar Ferraro

Jason Ferrell

Rodrigo Figueroa 
Bill Finch-Savage

Albert Fischer

Michael Luke Flessner

Michael E. Foley

Frank Forcella

Antonio Franco

Andrew Friskop

George Frisvold

E. Patrick Fuerst

Todd A. Gaines

Robert Sean Gallagher

Eric R. Gallandt

Melinda A. Gammon

Travis W. Gannon

John Gaskin

David Gealy

Roland Gerhards

Lyn Gettys

Claudio Marco Ghersa

Darci Giacomini

Kevin D. Gibson

Gurjeet S. Gill

Thomas K. Gitsopoulos

Rakesh Kumar Godara

Yaakov Goldwasser

Robin Gomez

Mustapha Gorai

Greta Gramig

Fabio Gresta

W. James Grichar

James L. Griffin

Arthur Grube

Andrea C. Grundy

Jeffrey L. Gunsolus

Aaron G. Hager

Bradley D. Hanson

Stuart Patrick Hardegree

Chrisopher Hardy

K. Neil Harker

Timothy Brian Harrington

Harlene Mae Hatterman-Valenti

Ian Heap

A. Heggenstaller

Michal Hejcman

Juan Manuel Herrera

Melissa J. Hills

Jodie S. Holt

Alois Honek

David P. Horvath

Anikó Horváth

Jared Adam Hoyle

Pierre Hucl

Cynthia Huebner

Andrew G. Hulting

Lynn Ingegneri

James Stuart Jacobs

Marie A. Jasieniuk
Prashant Jha

Amit J. Jhala

William G. Johnson

David L. Jordan

Mithila Jugulam

Miroslav Jursík

Laila Monika Karlsson

Shiv Kaundun

George Ogada Kegode

Karen Kennedy

Jon King

Andrew Robert Kniss

Suk Jin Koo

Nicholas E. Korres

Hansjoerg Kraehmer

Per Kudsk

Yong In Kuk

Sarah Lancaster

Bridget Robinson Lassiter

Ryan M. Lee

Deirdre Lemerle

Ramon G. Leon

Xiao Li

Matt Liebman

Ginger G. Light

Richard Bradley Lindenmayer

Rick S. Llewellyn

Hsu LM

Martin Anderson Locke

Louis Longchamps

Mark M. Loux

Stella Lovelli

Jon Lundgren

Mark Lundy

Rodney Lym

Drew Lyon

Kelly G. Lyons

Rong $\mathrm{Ma}$

Gregory MacDonald

John D. Madsen

Gulshan Mahajan

Mayank S. Malik

Carol A. Mallory-Smith

M. A. Susan Marles

Michelle C. Marra

Michael W. Marshall

Zdenka Martinkova

John Masiunas

Dario Massa

Joseph H. Massey

Hannah Mary Mathers

Bruce D. Maxwell

Robert J. Mayer

Lambert B. McCarty

William B. McCloskey

Patrick E. McCullough

James D. McCurdy 
Joseph Scott McElroy

Patrick McMullan

Bo Melander

Fabian Daniel Menalled

Mohsen Beheshtian Mesgaran

Stephen L. Meyers

Menghe Miao

Albrecht Michel

Lindsey Milbrath

Timothy W. Miller

Steven B. Mirsky

Paul Mitchell

Jorgelina Ceferina Montoya

Peter Morrell

John Bradley Morris

David Mortensen

Chris Mudge

Thomas C. Mueller

George W. Mueller-Warrant

Joseph C. Neal

Michael D. Netherland

Paul Neve

Mathieu Ngouajio

Robert L. Nichols

Yuki Niinomi

Scott Jay Nissen

Jason K. Norsworthy

James Oard

Dennis Calvin Odero

Junichi Okuno

Micheal D. K. Owen

Fernando Lania Pagbilao

David Pannell

Aaron Patton

William Louis Patzoldt

Ronald Edward Peachey

Sally Peltzer Peltzer

Alejandro Perez-Jones

Lora B. Perkins

Dallas E. Peterson

Ronald Pierik

Angela R. Post

Karina Potter

Lauren Poulos

Stephen B. Powles

Timothy S. Prather

Andrew Jennings Price

Atul Puri

Petr Pysek

Irena Rajcan

Neha Rana

Corey V. Ransom

Chittaranjan Ray

Chris Reberg-Horton

Jordi Recasens

Jacob Reed

Emilie E. Regnier
Karen A. Renner

Mark J. Renz

Dilpreet Singh Riar

Daniela Ribeiro

Claire Richard

Melissa Riley

Matt Rinella

Christopher Rosinger

Baruch Rubin

Matthew Ryan

Robert Douglas Sammons

Bielinski M. Santos

Norbert Satchivi

Maurizio Sattin

Laura Scarabel

Peter Scharf

Brian E Scheffler

Jill Schroeder

Brian J. Schutte

Steven S. Seefeldt

Brent A. Sellers

Scott A. Senseman

Yasser M. Shabana

Dale L. Shaner

David R. Shaw

Andy W. Sheppard

S. J. Shirtliffe

Vinod K. Shivrain

Eli Shlevin

Douglas Edward Shoup

Anil Shrestha

Peter H. Sikkema

Nerka Silingis

Marie-Josee Simard

Chris Sinclair

Brian M. Sindel

Samunder Singh

Joshua Skelton

Reid J. Smeda

Richard G. Smith

Upender R. Somireddy

Zhizhong Song

Lynn Sosnoskie

Josef Soukup

Kurt Spokas

Christy L. Sprague

Kathryn Steadman

Lawrence E. Steckel

Daniel O. Stephenson, IV

Neal Stewart

John Stinchombe

David E. Stoltenberg

Robert Stougaard

Clarence J. Swanton

François J. Tardif

John Teasdale

Angela Tellez-Rio 


\author{
David Thornby \\ Joel Torra \\ Joel Torra Farré \\ Hilmi Torun \\ Eric Tozzi \\ Ilias S. Travlos \\ Shane Turner \\ Hiroshi Uchino \\ Mahesh K. Upadhyaya \\ Rene Van Acker \\ Marcel van der Heijden \\ Laura Carol Van Riper \\ Johannes van Staden \\ Mark VanGessel \\ Vijay Varanasi \\ Martin Miguel Vila-Aiub \\ Eric Visser \\ Dean S. Volenberg \\ George Vourlitis \\ Maurizio Vurro \\ Michael Walsh \\ Guang-Xi Wang \\ Sarah Ward \\ Mark A. Weaver \\ Glenn Wehtje
}

Martin Weis

Stephen C. Weller

Rodrigo Werle

Paula Westerman

Leslie Ann Weston

James H. Westwood

Timothy Lee Widmer

Christian J. Willenborg

Bill J. Williams

Sam E. Wortman

Marcos Ezequiel Yanniccari

Fred H. Yelverton

Joe Yenish

Melinda Yerka

Carla Yerkes

Yasuyuki Yoshimura

Bryan G. Young

Qin $\mathrm{Yu}$

Lewis H. Ziska

Richard Zollinger

\section{Literature Cited}

2014 Journal Citation Reports (2015) 2014 Journal Citation Reports, Science Edition. New York: Thomas Reuters. 